\title{
THE DARK SIDE OF GLOBULAR CLUSTERS
}

\author{
P.-Y. LONGARETTI \\ Observatoire de Grenoble \\ BP $53 X 38041$ Grenoble Cedex 9 - FRANCE
}

AND

R. TAILLET, P. SALATI

$L A P P$

B.P. 110, 74941 Annecy-le-Vieux - FRANCE

\section{Objectives and method}

Searches of low-mass stars have become possible in globular clusters, and the first results suggest that the mass function turns up below $\sim 0.4 \mathrm{M}_{\odot}$ (Fahlman et al. 1989; Richer et al., 1990; Richer et al. 1991; G. Piotto, these proceedings). This conclusion is independently supported by the nonparametric dynamical analysis of four clusters by Gebhardt and Fisher 1995.

We have tried to explore how much mass can be hidden in globular clusters in the form of low mass stars and brown dwarves without violating the known observational constraints, and to devise observational strategies to uncover their presence. To this effect, we have constructed series of multi-mass King models of globular clusters with fixed mass function between 0.2 and $1.3 \mathrm{M}_{\odot}$ (the observationally constrained mass range), and varying power-law mass function below $0.2 \mathrm{M}_{\odot}$, down to some arbitrary cutoff. The ratio of light to massive star masses varies between 0 and 10 in these models. For definiteness, all our models have the same central surface brightness in the $\mathrm{V}$ band $\left(10000 \mathrm{~L}_{\odot} / \mathrm{pc}^{2}\right)$, the same total luminosity $(3 \times$ $\left.10^{5} \mathrm{~L}_{\odot}\right)$, and dispersion parameter for the red giants $(7 \mathrm{~km} / \mathrm{s})$. The mass to luminosity relations are taken from D'antona 1987 and Burrows et al. 1993, except for the red giant bin where it is assumed to be equal to 10 $\mathrm{L}_{\odot} / \mathrm{M}_{\odot}$ (Lupton et al. 1987). Finally, the squares of the velocity dispersion parameters in each mass bin are assumed to be inversely proportional to the mass. This assumption is commonly made in dynamical analyses 
of globular cluster data, and is partially supported by the rapidity of the thermalization process in cluster cores. However, thermalization is at best incomplete in the outskirsts of globular clusters, so that this approximation maximizes the quantity of low mass stars which can be hidden in globular clusters for a given set of observational constraints.

\section{Conclusions:}

Complete results obtained from this modelling can be found in Taillet et al. $1995 \mathrm{a} \& \mathrm{~b}$. The most interesting conclusions are the following:

Evaporation is too inefficient to eject low-mass stars from globular clusters. However, whether low-mass stars can survive gravitational shocking remains to be seen.

Luminosity profiles are basically unaffected by the presence of low-mass stars over about three decades in luminosity. On the other hand, velocity dispersion profiles flatten out when low-mass stars are added; unfortunately, these profiles are at present too poorly determined to be discriminant.

Thermalisation tends to confine heavy stars in the inner parts of the cluster and low-mass stars in the outskirts. These low-mass stars could be detected there in the infrared by ISO or SIRTF if they constitute more than half the cluster mass and if the thermalisation process is efficient enough.

Low-mass stars can also induce several microlensing events per year on background stars. Some clusters are present in the observation windows of the OGLE collaboration, but the statistics are too scarce to draw any conclusion at the moment.

\section{References}

Burrows, A. Hubbard, W. B., Saumon, D., and Lunine, J. I., 1993, ApJ, 406, 158.

D'Antona, F. 1987, ApJ, 320, 653.

Fahlman, G. G., Richer, H. B., Searle, L. and Thompson, I. B. 1989, ApJ, 343, L49.

Gebhardt, K., and Fischer, P. 1995, AJ, 109, 209.

Lupton, R. H., Gunn, J. E., and Griffin, R. F., 1987, AJ, 93, 1114.

Richer, H. B., Fahlman, G. G., Buonanno, R., and Fusi Pecci, 1990, ApJ, 359, L11.

Richer, H. B., Fahlman, G. G., Buonanno, R., Fusi Pecci, Searle, L., and Thompson, I. B., 1991, ApJ, 381, 147.

Taillet, R., Longaretti, P.-Y., and Salati, P. 1995a, To be published in Astropart. Phys.

Taillet, R., Salati, P. and Longaretti, P.-Y. 1995a, To be published in ApJ. 